\title{
Determinants of the Change in Arterial Stiffness in Peritoneal Dialysis Patients
} A Cross-Sectional and Longitudinal Study after Initiation of Therapy

\author{
Mitsutaka Nakahigashi, ${ }^{1} \mathrm{MD}$, Hiroyasu Tsukaguchi, ${ }^{1} \mathrm{MD}$, Satoshi Morimoto, ${ }^{2} \mathrm{MD}$, \\ Chikara Nakano, ${ }^{1}$ MD, Hiroko Ueda, ${ }^{1}$ MD, Kazunori Someya, ${ }^{1}$ MD, Makiko Kusabe, ${ }^{1}$ MD, \\ Sanae Kikuchi, ${ }^{1} \mathrm{MD}$, Takanobu Imada, ${ }^{1} \mathrm{MD}$ and Ichiro Shiojima, ${ }^{1} \mathrm{MD}$
}

\begin{abstract}
Summary
Arterial stiffness is an important risk factor for cardiovascular disease (CVD) in patients with end-stage renal failure. However, little is known about the factors that contribute to arterial rigidity in peritoneal dialysis (PD) patients. The aim of this study was to define the pattern and determinants of the longitudinal change in arterial stiffness after PD initiation.

Arterial stiffening was estimated for 46 PD patients by using brachial-ankle pulse wave velocity (baPWV) and carotid intima-media thickness (cIMT). The cross-sectional relationship between the arterial markers and their clinical determinants was studied. The longitudinal effects of blood pressure (BP), body fluid status, and glucose were studied over the two years after initiating PD.

Multivariate analysis showed that higher baPWV was associated positively with urinary protein excretion $(P<0.001)$, systolic BP $(P=0.001)$, and hemoglobin A1c $(P=0.003)$. In contrast, increased cIMT correlated with smoking $(P=0.004)$ and hypoalbuminemia $(P=0.04)$, suggesting that endothelial dysfunction is implicated in the atherogenic process. Neither cIMT nor baPWV correlated significantly with other PD-related covariates of volume overload, peritoneal solute transport, kidney function, and C-reactive protein. Longitudinal observation demonstrated that BP had a greater influence on baPWV changes than hyperglycemia or fluid status.

Our study indicates that 1) baPWV represent an arterial marker that integrates multifactorial interaction between modifiable variables including BP and plasma glucose; and 2) intervention aimed at controlling BP as well as nutritional conditions (glucose and albumin) may reduce CVD risk in PD patients.
\end{abstract}

(Int Heart J 2017; 58: 915-925)

Key words: Cardiovascular disease, Pulse wave velocity, Intima-media thickening, End stage kidney disease

A rterial stiffness is an important risk factor for cardiovascular disease (CVD) in patients with endstage kidney disease (ESKD). ${ }^{1}$ Multiple factors contribute to the stiffening process, including hemodynamics, nutrition, and inflammation. ${ }^{2)}$ Early recognition and modification of specific factors that rigidify arteries help prevent CVD. Non-invasive vascular testing, pulse wave velocity (PWV), and carotid intima-media thickness (cIMT) are increasingly used as surrogate biomarkers of CVD. ${ }^{3)} \mathrm{PWV}$ is the most widely used measure of arterial stiffening in patients at higher CVD risk. ${ }^{4)}$ The advantage of PWV is its ability to reflect reversible, functional vascular rigidity, and thereby serve as a marker of therapeutic responsiveness.5) On the other hand, cIMT represents a morphological change that reflects not only early atherosclerosis, but also non-atherosclerotic remodeling with medial hypertrophy. An increase in cIMT is a predictive marker of CVD. ${ }^{6}$ Many studies in CKD and ESKD patients have demonstrated that increased PWV and cIMT are associated with an increased incidence of CVD. ${ }^{7)}$ In hemodialysis patients, raised PWV predicts both CVDrelated and overall mortality. However, relatively few studies have evaluated the utility of arterial stiffness in patients on peritoneal dialysis (PD). Arterial stiffness in PD patients correlated with the increase in body fluid volume or peritoneal transport rate ${ }^{8,9)}$ and was an independent predictor of cardiovascular events. ${ }^{10)}$

The aim of this study was to define the determinants

From the ${ }^{1}$ Department of Medicine II, Kansai Medical University, Hirakata, Japan and ${ }^{2}$ Department of Medicine II, Endocrinology and Hypertension, Tokyo Women's Medical University, Tokyo, Japan.

This work was supported by research grants from the Japan Society for the Promotion of Science (Grant-in-Aid for Scientific Research: C 26461246, and Grant-in-Aid for Exploratory Research: 2465950) and the Osaka Kidney Foundation to H.T. M.N was supported by a young investigator award from Kansai Medical University.

Address for correspondence: Hiroyasu Tsukaguchi, MD, Department of Medicine II, Kansai Medical University, 2-5-1 Shinmachi, Hirakata, Osaka 5731010, Japan. E-mail: tsukaguh@hirakata.kmu.ac.jp

Received for publication December 14, 2016. Revised and accepted February 10, 2017.

Released in advance online on J-STAGE November 17, 2017.

doi: 10.1536/ihj.16-624

All rights reserved by the International Heart Journal Association. 


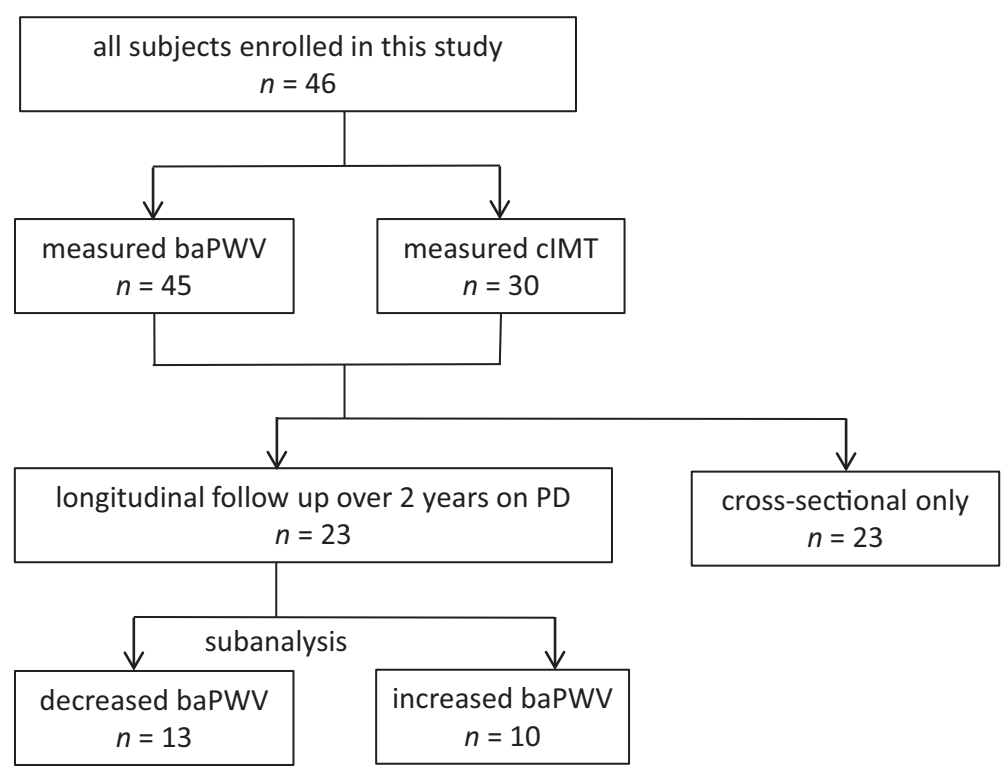

Figure 1. Study design of patient enrollment and flowchart showing the sub-group analysis. Forty-five of the $46 \mathrm{PD}$ patients were evaluated for baPWV, whereas 30 were assessed for cIMT. Then, 23 of the 46 patients underwent repeated measurements of baPWV and its associated clinical variables including $\mathrm{BP}$ and $\mathrm{HbA} 1 \mathrm{c}$ over two years following the commencement of PD. Based on the baPWV values during the PD therapy at three distinct time points (pre-PD and at the 1st and the 2nd PD year), the patients were divided into two subgroups based upon the changes in baPWV: one with a gradual rise in baPWV $(n=10)$, and the other with a lowering baPWV value over the first PD year $(n=13)$. PD indicates peritoneal dialysis; baPWV, brachial-ankle pulse wave velocity; and cIMT, carotid intima media thickness.

of arterial stiffening in PD patients, particularly in the first two years after initiation of PD by means of cIMT and brachial-ankle PWV (baPWV). BaPWV involves determining the velocity of pulse propagation between the brachial and ankle that reflects vascular stiffness of both peripheral muscular conduit arteries and central elastic, capacitive vessels. ${ }^{11)}$ In PD patients, there are relatively limited data on baPWV compared with data on carotidfemoral PWV (cfPWV), a well-established index of central arterial stiffness. ${ }^{12)}$ However, baPWV has several advantages for risk assessment. First, baPWV can be performed easily and routinely as it only requires wrapping the four extremities with blood pressure cuffs. ${ }^{13)}$ Second, there is ample reference data for baPWV from previous epidemiological studies. ${ }^{14)}$ Third, baPWV is better for evaluating the rigidity of muscular-type arteries in addition to that of central elastic arteries. Fourth, previous studies on populations at higher risk of CVD have demonstrated that elevated baPWV predicts not only vascular damage (e.g., micro-albuminuria), but also disease end points such as coronary events and stroke. ${ }^{11)}$

Our multivariate analyses showed that baPWV correlated positively with urinary protein excretion, systolic blood pressure (BP), and plasma glucose. We first demonstrated that baPWV showed a temporal attenuation in accordance with the concomitant decline of BP over the first PD years. Hemodynamic factors had the greatest impact on baPWV, while metabolic milieu (i.e., glucose and/or malnutrition) also contributed to increasing baPWV. In contrast, cIMT was correlated distinctive factors of smoking and hypoalbuminemia, suggesting an essential role of endothelial dysfunction in cIMT changes after starting PD.

\section{Methods}

Subjects and biochemical analysis: This was a prospective, cross-sectional, single-center study. Forty-six PD patients (26 male, 20 female, mean age 55 years) who visited our hospital between January 2009 and March 2013 were enrolled (Figure 1, Table I). The study protocol was reviewed and approved by the Ethics Committee of Kansai Medical University.

The duration of PD was 24.6 months (range, 2-87 months). The patients were treated using either an automated device (APD, $45 \%$ ), or continuous ambulatory peritoneal dialysis (CAPD, 55\%). Standard, neutral $1.25 \%$ glucose-based or/and icodextrin (Baxter Healthcare, Tokyo, Japan) dialysates were used. Plasma glucose was measured at arbitrary time on clinical visit. The adequacy of dialysis $(\mathrm{Kt} / \mathrm{V})$ was calculated using a singlecompartment model. Trans-peritoneal solute transport rate between dialysate and plasma $(\mathrm{D} / \mathrm{P})$ was estimated by a 4 hour equilibration test.

Measurement of baPWV: BaPWV was measured using an automatic oscillometry device (BP203RPE II, Omron Colin) before and every six months after initiating PD. After a 5-minute rest in the supine position, cuffs were 
Table I. Clinical Characteristics of the PD Patients

\begin{tabular}{|c|c|}
\hline Parameters & $n=46$ \\
\hline Age (years) & $55 \pm 14$ \\
\hline Dialysis duration (months) & $24.6(2-87)$ \\
\hline Gender (Male \%) & 57 \\
\hline \multicolumn{2}{|l|}{ Smoking habit } \\
\hline Current (\%) & 26 \\
\hline Past (\%) & 22 \\
\hline Brinkman index* & $494(0-3800)$ \\
\hline \multicolumn{2}{|l|}{ Etiology of ESRD } \\
\hline Diabetes $(\%)$ & 37 \\
\hline Glomerulonephritis (\%) & 32 \\
\hline Hypertension $(\%)$ & 22 \\
\hline Polycystic kidney disease (\%) & 9 \\
\hline \multicolumn{2}{|l|}{ Cardiovascular } \\
\hline Body mass index $\left(\mathrm{kg} / \mathrm{m}^{2}\right)$ & $23.5 \pm 3.7$ \\
\hline LVM index $\left(\mathrm{g} / \mathrm{m}^{2}\right)$ & $102.7 \pm 33.2$ \\
\hline $\operatorname{LVEF}(\%)$ & $64 \pm 12$ \\
\hline \multicolumn{2}{|l|}{ Blood pressure (mmHg) } \\
\hline Systolic & $141 \pm 24$ \\
\hline Diastolic & $83 \pm 14$ \\
\hline Mean arterial & $103 \pm 17$ \\
\hline Pulse pressure & $57 \pm 15$ \\
\hline Heart rate (beats/minute) & $76 \pm 14$ \\
\hline \multicolumn{2}{|l|}{ Medications } \\
\hline Erythropoiesis stimulating agents (\%) & 96 \\
\hline RAS blockades $(\%)$ & 89 \\
\hline Beta-blockers $(\%)$ & 37 \\
\hline $\mathrm{Ca}$ antagonists $(\%)$ & 78 \\
\hline Anti-platelet agents (\%) & 30 \\
\hline Statins $(\%)$ & 26 \\
\hline \multicolumn{2}{|l|}{ PD-related variable } \\
\hline Residual renal weekly Kt/V & $0.54 \pm 0.51$ \\
\hline Fluid removal $(\mathrm{mL})$ & $800(-600-3511)$ \\
\hline Total weekly Kt/V & $1.9 \pm 0.8$ \\
\hline \multicolumn{2}{|l|}{ Non-invasive arterial biomarkers } \\
\hline $\mathrm{baPWV}(\mathrm{cm} / \mathrm{s})$ & $1764 \pm 429$ \\
\hline $\operatorname{cIMT}(\mathrm{mm})$ & $0.89 \pm 0.51$ \\
\hline \multicolumn{2}{|l|}{ Body fluid status } \\
\hline ECW/TBW & $0.40 \pm 0.01$ \\
\hline
\end{tabular}

Data are shown as mean \pm SD or number (\%). PD indicates peritoneal dialysis; ESRD, end stage renal disease; ADPKD, autosomal dominant polycystic kidney disease; LVM, left ventricular mass; LVEF, left ventricular ejection fraction; RAS, renin-angiotensin system; PD, peritoneal dialysis; D/P $\mathrm{Cr}$, dialysate/plasma creatinine; baPWV, brachial-ankle pulse wave velocity; cIMT, carotid intima-media thickness; and ECW/TBW, extracellular water/total body water. *Brinkman index was obtained by multiplying numbers of cigarettes (per day) by smoking duration (years).

applied onto both arms and ankles. Pulse pressure waveforms of the brachial and tibial arteries were captured simultaneously, followed by measurement of the time interval between the initial peaks of these waveforms (Tba). The PWV was calculated according to the previous literature. $^{13,15)}$ Patients with an ankle-brachial index $(\mathrm{ABI})<0.9$ were excluded from further evaluations.

Measurement of cIMT: cIMT was measured by B-mode ultrasonography using a $5 \mathrm{MHz}$ linear transducer (a10 color Doppler system, Aloka). The thickness of the carotid artery walls was measured on straight longitudinal $10 \mathrm{~mm}$ segments of the common carotid artery at positions 2-3 cm apart from the carotid bulb. ${ }^{6,16)}$ The thickness was evaluated in arterial segments free of plaques and was defined as the distance between the leading edge of the most internal echogenic line (lumen-intima interface) and the second echogenic line (media-adventitia interface).

Measurement of body fluid volume: Multi-frequency segmental bioelectrical impedance was measured by using the InBody $720 .{ }^{17)}$ To estimate a body fluid volume accurately, extracellular water $(\mathrm{ECW})$ was corrected by total body water (TBW), yielding the ratio of ECW/TBW.

Statistical analysis: Comparison between two independent groups was performed with un-paired Student's t-test. A pair-wise correlation between continuous variables was analyzed by Pearson's correlation test. Uni- and multivariate analyses were performed by SPSS version 20.0 (IBM, Armonk, NY).

\section{Results}

Characteristics of PD patients: A total of 46 patients were studied, of whom $37 \%$ had diabetes mellitus (Table I and Table II). The vast majority (44 of 46) had hypertension and were taking anti-hypertensive medication. Hemoglobin levels were controlled to a mean of $10.6 \mathrm{~g} / \mathrm{dL}$ with most patients $(96 \%)$ being treated with erythropoiesis stimulating agents (Table I). Mean glucose and serum lipid levels remained within the recommended ranges of hemoglobin A1c (HbA1c) 5.8\% and LDL cholesterol $101.4 \mathrm{mg} / \mathrm{dL}$. With regards to the morbidity and mortality during the observational period, three patients manifested the myocardial infarction but eventually recovered from it by medical and/or coronary intervention therapy. The other three individuals died from the septic shock, dissecting aneurysm, and constrictive pericarditis.

Cross-sectional covariates of PWV and cIMT: A series of measurements of baPWV and cIMT were obtained at subsequent arbitrary time-points (every 6 months for baPWV, and every 12 or 24 months for cIMT) (Figure 2). Univariate analysis showed that baPWV was associated with plasma glucose $(r=0.57, P<0.001)$, HbA1c $(r=$ $0.56, P<0.001)$, hypoalbuminemia $(r=-0.53, P<$ $0.001)$, and urinary protein excretion $(r=0.53, P<0.001)$ (Table III). There was also a modest but significant association between baPWV and increases in glycoalbumin $(r$ $=0.45, P=0.003)$, pulse pressure $(r=0.42, P=0.004)$, systolic BP $(r=0.37, P=0.01)$, and age $(r=0.34, P=$ $0.02)$, as well as lower LDL cholesterol $(r=-0.35, P=$ 0.02 ). However, the baPWV values did not correlate with cIMT or body-fluid overload (ECW/TBW, Table III). The PD-related parameters including adequacy of dialysis (Kt/ $\mathrm{V})$, kidney reserve, and peritoneal transport rate $(\mathrm{D} / \mathrm{P}) \mathrm{did}$ not show any association with baPWV (data not shown).

In contrast, cIMT showed a strong association with Brinkman index (cumulative cigarette consumption, $r=$ $0.71, P<0.001)$, diastolic BP $(r=-0.59, P=0.001)$ and mean arterial pressure $(r=-0.56, P=0.001)$ (Table III). Hypoalbuminemia $(r=-0.53, P=0.003$ ), high sensitivity C-reactive protein (hsCRP; $r=0.52, P=0.004$ ), and lower systolic BP $(r=-0.50, P=0.005)$ showed a significant but slightly weaker univariate association with cIMT. In addition to age $(r=0.46, P=0.01)$, other PD-related 
Table II. Biochemical Parameters of PD Patients

\begin{tabular}{|c|c|}
\hline Variables & $n=46$ \\
\hline LDL cholesterol (mg/dL) & $101.4 \pm 23.9$ \\
\hline HDL cholesterol (mg/dL) & $47.5 \pm 14.5$ \\
\hline Triglyceride (mg/dL) & $154.5 \pm 92.9$ \\
\hline Blood sugar (mg/dL) & $120.5 \pm 36.4$ \\
\hline Hemoglobin A1c $(\%)^{*}$ & $5.8 \pm 0.9$ \\
\hline Glycoalbumin (\%) & $14.5 \pm 3.1$ \\
\hline Calcium (mg/dL) & $9.5 \pm 0.7$ \\
\hline Phosphorus (mg/dL) & $5.8 \pm 1.2$ \\
\hline $\mathrm{Ca} \times \mathrm{P}\left(\mathrm{mg}^{2} / \mathrm{dL}^{2}\right)$ & $54.3 \pm 11.4$ \\
\hline Intact PTH (pg/mL) & $191.4(23.0-650.0)$ \\
\hline hsCRP (mg/dL) & $0.212(0.006-1.689)$ \\
\hline Hemoglobin (g/dL) & $10.6 \pm 1.1$ \\
\hline Total protein $(\mathrm{g} / \mathrm{dL})$ & $6.4 \pm 0.6$ \\
\hline Serum albumin (g/dL) & $3.4 \pm 0.6$ \\
\hline Blood urea nitrogen (mg/dL) & $56.4 \pm 16.9$ \\
\hline Creatinine $(\mathrm{mg} / \mathrm{dL})$ & $10.1 \pm 3.1$ \\
\hline Uric acid (mg/dL) & $6.7 \pm 1.1$ \\
\hline Serum beta 2 microglobulin (mg/dL) & $24.4 \pm 9.5$ \\
\hline $\mathrm{hANP}(\mathrm{pg} / \mathrm{mL})$ & $80.5 \pm 56.7$ \\
\hline NT-proBNP (pg/mL) & $8367.7(181.0-81248.0)$ \\
\hline Proteinuria (protein/creatinine ratio) $(\mathrm{mg} / \mathrm{gCr}$ ) & $4392.0(371.6-4392.0)$ \\
\hline
\end{tabular}

Data are shown as mean \pm SD with ranges (numbers in parenthesis). PTH indicates parathyroid hormone; hsCRP, high-sensitivity C-reactive protein; hANP, human atrial natriuretic peptide; and NT-proBNP, N-terminal probrain natriuretic peptide. *Hemoglobin A1c was indicated in a NGSP scale (normal range 4.6 to $6.2 \%$ ).

parameters, including phosphorus $(r=-0.45, P=0.01)$, calcium $(r=0.44, P=0.02)$, and $\mathrm{Ca} \times \mathrm{P}(r=-0.39, P=$ 0.04) showed a weak correlation with cIMT (Table III). Similar to baPWV values, PD duration, adequacy, solute transport rate $(\mathrm{D} / \mathrm{P})$, body-fluid volume (ECW/TBW), and residual kidney function did not have any significant influence on cIMT.

Given that $\mathrm{CV}$ risk factors in the elderly are usually multifactorial and mutually related, we next analyzed the correlation between the arterial markers in multivariate models. These analyses demonstrated that baPWV was associated independently with urinary protein excretion $\left(r^{2}=\right.$ $0.71, P<0.001)$, age $\left(r^{2}=0.71, P<0.001\right)$, systolic BP $\left(r^{2}=0.71, P=0.001\right)$, and HbA1c $\left(r^{2}=0.71, P=0.003\right)$, even after adjustment for serum albumin and LDL cholesterol (Table IV). Notably, the correlations with cIMT were distinct from those of baPWV, with Brinkman index $\left(r^{2}=\right.$ $0.59, P=0.004)$ and hypoalbuminemia $\left(r^{2}=0.59, P=\right.$ $0.04)$, showing significant correlation after adjustment for hsCRP and diastolic pressure (Table IV). The presence of distinctive correlates for cIMT, smoking and hypoalbuminemia, suggested that cIMT may reflect physiological processes slightly different from those involved in baPWV.

To define the role of glycemic control, we conducted a sub-analysis by dividing the patients into two groups of non-diabetes mellitus (DM) and DM (Figure 3). The results indicated that the DM group had a higher baPWV than the non-DM group (1973 versus $1638 \mathrm{~cm} / \mathrm{second}, P$ $=0.009)$. On the other hand, cIMT values did not differ between the non-DM and DM subgroups (0.94 versus $0.79 \mathrm{~mm}$ ). These results indicated that hemodynamic con- ditions are critical determinants of both baPWV and cIMT in PD patients. The association of baPWV with HbAlc and glycoalbumin suggested that multiple factors including hyperglycemia could affect arterial stiffness.

Longitudinal correlates of $\triangle$ baPWV: We next carried out a prospective investigation of the clinical covariates involved in changes in baPWV ( $\triangle \mathrm{baPWV}$ ) over the twoyear after PD initiation. The baPWV values reduced during the first year of PD (mean 1691 to $1627 \mathrm{~cm} / \mathrm{second}$ ) but did not reach statistical significance (data not shown). Conversely, there was an overall tendency for a subtle increment in baPWV during the second year of PD year (mean 1627 to $1728 \mathrm{~cm} / \mathrm{second}$ ). We next examined the longitudinal relationship between $\triangle \mathrm{baPWV}$ and three representative correlates, BP, body fluid status, and plasma glucose. In the univariate analysis, $\triangle$ baPWV showed a positive correlation with changes in systolic BP ( $\Delta$ systolic BP) in both first and second years after PD initiation ( $r=0.72, P<0.001$ versus $r=0.70, P=0.001$ (Figure 4 A). Similarly, $\Delta$ diastolic BP correlated positively with $\triangle \mathrm{baPWV}$ in both first and second years, albeit to a lesser degree than $\Delta$ systolic BP, $(r=0.53, P=0.009$ versus $r=$ $0.66, P=0.002$ ) (Figure 4B). These results indicated that $\Delta$ systolic BP had a stronger impact on $\Delta$ baPWV than $\Delta$ diastolic BP. Multivariate analysis showed that $\Delta$ systolic BP was the only independent determinant of $\triangle$ baPWV between pre-PD and the first PD year $\left(r^{2}=0.49, P<0.001\right.$, $n=23$ ) (Table V). Further analysis comparing the data between the first and second PD year showed that $\Delta$ systolic BP $\left(r^{2}=0.59, P=0.02, n=19\right)$ still remained an independent determinant of $\triangle \mathrm{baPWV}$, although the correlation became weaker during the second PD year (Table V). 
$\Delta$ baPWV $(n=23)$
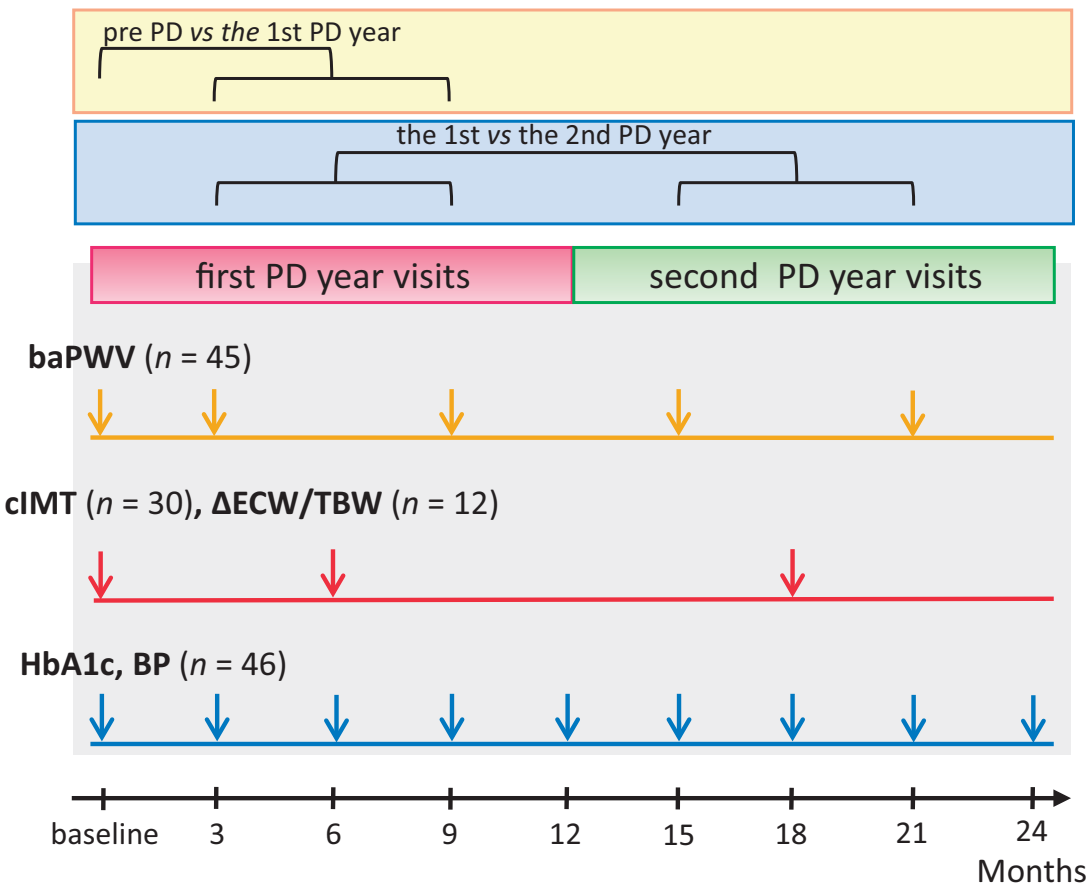

Figure 2. Follow-up examination protocol and scheme for clinical data collection. BaPWV, cIMT, $\triangle$ ECW/TBW were measured at baseline prior to PD (pre-PD) and were followed up at interval of twice (baPWV) or once a year (cIMT, $\triangle \mathrm{ECW} / \mathrm{TBW}$ ). Among the two baPWV values determined annually, only one which was obtained at time point closest to cIMT measurement, was used for further analysis. To assess changes in baPWV during the first PD year, the data between pre-PD base line and those at post-PD 2 to 12 months were compared ( $\triangle$ baPWV: pre-PD versus the first PD year). The $\triangle \mathrm{baPWV}$ in the second PD year was obtained by comparing the data between at 2 to 12 months and 13 to 24 months (the first versus second PD year). HbA1c and blood pressure were examined at every monthly visit. Vertical arrows in the upper panels indicate which datasets were used for longitudinal comparison in Figures and Tables. PD indicates peritoneal dialysis; BP, blood pressure; HbA1c, hemoglobin A1c; baPWV, brachial-ankle pulse wave velocity; cIMT, carotid intima media thickness; and ECW/TBW, extracellular water/total body water.

Changes in markers for body fluid status (i.e., $\triangle \mathrm{hANP}$, $\Delta$ body weight, and $\Delta \mathrm{ECW} / \mathrm{TBW}$ ) had no significant influence on baPWV values (data not shown). These results suggest that the observed $\Delta$ systolic BP may affect $\Delta$ baPWV through mechanisms other than volume expansion. Notably, HbA1c levels did not show a clear relationship with $\triangle$ baPWV during the first and second PD years (Figure 4C).

In order to further delineate the longitudinal relationship between BP and baPWV, we categorized the patients by dividing 23 patients into two subgroups, depending on the baPWV changes over time, either becoming higher or lower. Thirteen patients had a decreasing baPWV value during the first year of PD $(P<0.001)$ (Figure 1$)$. In all but one patient in this decreasing PWV group (12 in 13), both systolic and diastolic BP became lower in accordance with the decline in $\triangle$ baPWV $(P<0.001)$ (Figure 5 A-C). Despite the close relationship between baPWV and hemodynamic factors, $\triangle \mathrm{baPWV}$ did not correlate with HbA1c levels (Figure 5D). These data indicated the arterial rigidity-lowering effects are mainly related to the reduction of BP, rather than those of plasma glucose levels, particularly in the first year after starting PD.

\section{Discussion}

This is, to our knowledge, the first longitudinal study that clarifies the clinical correlates of baPWV overtime after initiation of PD. We chose a baPWV because of its technical simplicity only requires attaching an easy-to-use blood pressure cuff onto foot and arms and accumulating evidence for usefulness. ${ }^{11,13)}$ Most previous studies employed the femoral and heart (hfPWV) ${ }^{18)}$ or carotid (cfPWV), a well-established index of central arterial stiffness. ${ }^{19-21)}$ The cfPWV measures stiffness from the aorta to iliofemoral axes, while baPWV reflects one in not only central elastic arteries but also peripheral muscular arteries. Our 2-year follow-up observations indicated that BP is a prime determinant of baPWV: nearly half of our patients had a decreasing baPWV during the first PD year. Importantly, nearly all patients in this lowering baPWV subgroup showed a concomitant decrease in both systolic and diastolic BP after initiation of PD. Our study for the first time revealed that there is an overall decreasing ten- 
Table III. Univariate Association of Clinical Factors with baPWV or cIMT

\begin{tabular}{|c|c|c|c|c|}
\hline \multirow[t]{2}{*}{ Variable } & \multicolumn{2}{|c|}{$\begin{array}{l}\text { Dependent variable: } \\
\text { baPWV }(n=45)\end{array}$} & \multicolumn{2}{|c|}{$\begin{array}{l}\text { Dependent variable: } \\
\text { cIMT }(n=30)\end{array}$} \\
\hline & $r$ & $P$ & $r$ & $P$ \\
\hline Age & 0.34 & $0.02 *$ & 0.46 & $0.01 *$ \\
\hline Smoking habit & 0.40 & $0.01 *$ & 0.23 & 0.22 \\
\hline Brinkman index & 0.38 & $0.02 *$ & 0.71 & $<0.001 * *$ \\
\hline \multicolumn{5}{|l|}{ Cardiologic parameters } \\
\hline LVM index & 0.18 & 0.29 & -0.29 & 0.15 \\
\hline LVEF & 0.20 & 0.21 & -0.05 & 0.80 \\
\hline Systolic blood pressure & 0.37 & $0.01^{*}$ & -0.50 & $0.005^{*}$ \\
\hline Diastolic blood pressure & 0.16 & 0.30 & -0.59 & $0.001 * *$ \\
\hline Mean arterial pressure & 0.27 & 0.07 & -0.56 & $0.001 * *$ \\
\hline Pulse pressure & 0.42 & $0.004 * *$ & -0.32 & 0.09 \\
\hline Heart rate & 0.22 & 0.15 & 0.15 & 0.44 \\
\hline \multicolumn{5}{|l|}{ Biochemical parameters } \\
\hline LDL cholesterol & -0.35 & $0.02 *$ & -0.06 & 0.75 \\
\hline HDL cholesterol & 0.02 & 0.92 & -0.03 & 0.87 \\
\hline Triglyceride & -0.04 & 0.81 & -0.18 & 0.35 \\
\hline Glucose & 0.57 & $<0.001 * *$ & -0.18 & 0.35 \\
\hline Hemoglobin A1c & 0.56 & $<0.001 * *$ & -0.02 & 0.91 \\
\hline Glycoalbumin & 0.45 & $0.003 * *$ & 0.12 & 0.55 \\
\hline Calcium & 0.10 & 0.53 & 0.44 & $0.02 *$ \\
\hline Phosphorus & -0.02 & 0.92 & -0.45 & $0.01 *$ \\
\hline $\mathrm{Ca} \times \mathrm{P}$ & 0.02 & 0.89 & -0.39 & $0.04 *$ \\
\hline Intact PTH & 0.02 & 0.90 & -0.17 & 0.38 \\
\hline hsCRP & 0.26 & 0.09 & 0.52 & $0.004 * *$ \\
\hline Hemoglobin & -0.13 & 0.41 & -0.08 & 0.67 \\
\hline Total protein & -0.24 & 0.12 & -0.26 & 0.17 \\
\hline Serum albumin & -0.53 & $<0.001 * *$ & -0.53 & $0.003^{* *}$ \\
\hline Uric acid & -0.06 & 0.71 & -0.31 & 0.09 \\
\hline Serum beta 2 microglobulin & 0.09 & 0.60 & 0.05 & 0.81 \\
\hline hANP & 0.10 & 0.59 & 0.07 & 0.74 \\
\hline NT-proBNP & 0.11 & 0.53 & -0.03 & 0.89 \\
\hline Proteinuria (protein/creatinine ratio) & 0.53 & $<0.001 * *$ & -0.12 & 0.58 \\
\hline \multicolumn{5}{|l|}{ Body fluid status } \\
\hline ECW/TBW & 0.18 & 0.32 & 0.05 & 0.81 \\
\hline cIMT & 0.07 & 0.71 & & \\
\hline
\end{tabular}

Correlations were shown with co-efficient $(r)$ and $P$ values $(P)$ by use of Pearson's formula. LVM indicates left ventricular mass; LVEF, left ventricular ejection fraction; PTH, parathyroid hormone; hsCRP, high-sensitivity C-reactive protein; hANP, human atrial natriuretic peptide; NT-proBNP, N-terminal pro-brain natriuretic peptide; baPWV, brachialankle pulse wave velocity; cIMT, carotid intima-media thickness; and ECW/TBW, extracellular water/total body water. $* P<0.05, * * P<0.005$.

dency in PWV over the first PD year. The stiffnesslowering effects have been missed in previous longitudinal studies, ${ }^{18-21)}$ because the PWV was measured at two arbitrary time points in the maintenance phase when approximately 6 months passed after initiating PD. Then, we found that baPWV exhibited a trend of increasing over the second PD year in parallel with the gradual rise in BP. Notably, we did not see any correlation between changes in baPWV and HbA1c or body-fluid overload ( $\triangle \mathrm{ECW} /$ TBW) during PD, consistent with the observations by others. ${ }^{18-21)}$ Moreover, there was no relationship between changes in baPWV and serum calcium and phosphorus levels. These results together suggested that BP control is the first-line approach to retard or even reverse vascular stiffness in the first few years after introducing PD.

From a cross-sectional point of view, ${ }^{8,9)}$ volume overload has been reportedly shown to associate with cfPWV.
However, we did not find any relationship between baPWV and body-fluid overload cross-sectionally. This disparity might be due to the variability in peritoneal functions among the patients. One previous study demonstrated that both body-fluid overload (higher ECW/TBW) and faster solute transport (higher $\mathrm{D} / \mathrm{P}$ ) were independent determinants of higher cfPWV. ${ }^{9)}$ This suggests that these patients might be prone to develop volume overloaded partly because of the greater transmesothelial loss of osmotic agents, thereby losing the driving force sufficient for body fluid removal. In this context, our patients preserved an average level of peritoneal solute transport rate (mean $\mathrm{D} / \mathrm{P}=0.66$ ), which allowed them to maintain nearly normovolemia (mean ECW/TBW 0.40). Alternatively, the discrepant observations may be ascribed to the methodological reason: baPWV measures the stiffness in both muscular conduit vessels and central elastic capaci- 
Table IV. Multivariate Correlates of baPWV or cIMT

\begin{tabular}{lccc}
\multicolumn{1}{c}{ Variable } & $\beta$ coefficient & Standard error & $P$ \\
\hline Factors associating with baPWV & & & \\
Proteinuria (protein/creatinine ratio) & 0.51 & 0.01 & $<0.001$ \\
Age & 0.48 & 2.92 & $<0.001$ \\
Systolic blood pressure & 0.34 & 1.85 & 0.001 \\
Hemoglobin A1c & 0.31 & 47.63 & 0.003 \\
Factors associating with cIMT & & & \\
Brinkman index & 0.51 & $<0.001$ & 0.004 \\
Serum albumin & -0.35 & 0.16 & 0.04 \\
\hline
\end{tabular}

Independent determinants for greater baPWV were assessed by multi-variate analyses with adjustment of serum albumin and LDL cholesterol. The adjusted $r^{2}$ was 0.71 . Independent determinants for higher cIMT was assessed by multi-variate analyses with adjustments of hsCRP and diastolic blood pressure. The adjusted $r^{2}$ was 0.59 . Brinkman index was calculated by multiplying numbers of daily cigarette consumption and smoking duration (years). baPWV indicates brachial-ankle pulse wave velocity; cIMT, carotid intima-media thickness; and hsCRP, high-sensitivity C-reactive protein.

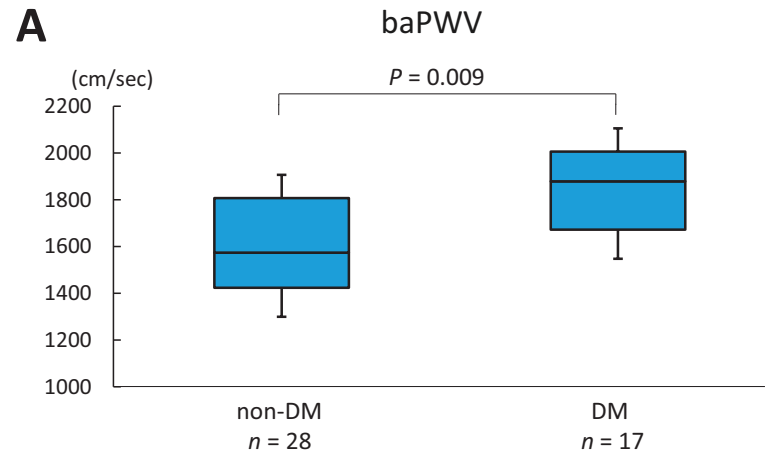

B

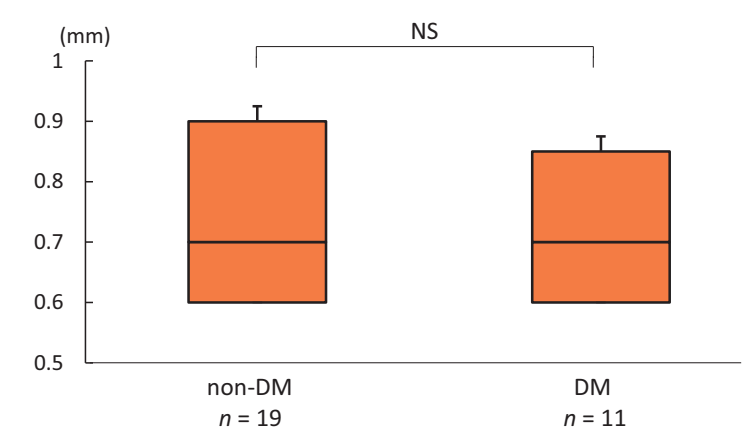

Figure 3. Comparison of baPWV and cIMT in non-diabetic and diabetic subgroups. The baPWV values were significantly higher in the diabetes mellitus (DM) group than in the non-DM group (1973 versus $1638 \mathrm{~cm} /$ second, $P=0.009$ ). On the other hand, cIMT values did not differ between the non-DM and DM groups $(0.94$ versus $0.79 \mathrm{~mm})$. DM indicates diabetes mellitus; baPWV, brachial-ankle pulse wave velocity; cIMT, carotid intima-media thickness; and NS, not significant.

tive arteries, while cfPWV mostly reflected central arterial rigidity. ${ }^{22)}$ The other important point in our cross-sectional analysis is that proteinuria was associated independently with faster baPWV in our PD patients. To our knowledge, proteinuria has not yet been previously reported as covariates of baPWV in PD patients. Vascular stiffness is shown to correlate with proteinuria in hypertensive and hyperglycemic patients. ${ }^{11)}$ It is therefore speculated that microvascular endothelial damage (proteinuria) and functional arterial stiffness (PWV) may share similar pathogenic mechanisms.

As consistent with our observations, most previous studies with assessment of arterial stiffness in either PD or HD patients failed to see any positive correlation between LDL and PWV values. ${ }^{23)}$ We consider that this lack of correlation may reflect the general tendency towards lower cholesterol levels in ESRD individuals. The dyslipidemia is probably thought to arise from the malnutrition and chronic inflammation, the major consequence of chronic uremic status, ${ }^{7)}$ but not from the difference in the dialysis modality. In fact, our PD patients showed, on average, normal LDL cholesterol (101.4 mg/dL) and slightly low serum albumin levels $(3.4 \mathrm{~g} / \mathrm{dL})$. Because of the uremiarelated lower LDL state, it appears that the blood pressure and glycemic levels are more relevantly associated with arterial stiffness than LDL levels, particularly in the earlier period after PD or HD introduction. Further study with larger cohort and longer follow-up is warranted to assess the role of dyslipidemia in progression of arterial stiffening.

We found different clinical determinants existed for baPWV and cIMT. Higher baPWV correlated positively with hyperglycemia and proteinuria, in addition to elevated systolic BP, whereas increased cIMT was associated with smoking and hypoalbuminemia. In our crosssectional analysis (Table IV), hyperglycemia contributed to baPWV but not cIMT. One possibility of the distinctive determinants for baPWV and cIMT could be ascribed to the different anatomic and physiological properties of arterial trees. Arterial stiffness results from complex interactions involving distensibility, compliance, and elastic modulus. Such properties are not uniform along the arterial tree. The baPWV measures stiffness, not only in the central elastic portion, but also in peripheral musculartype artery. The latter contains more abundant vascular smooth muscle cells. The baPWV in smaller caliber arteries may therefore be susceptible to even subtle delay, distorted microcirculation, or metabolic imbalances (i.e., high glucose levels). We therefore speculate that hypertension 

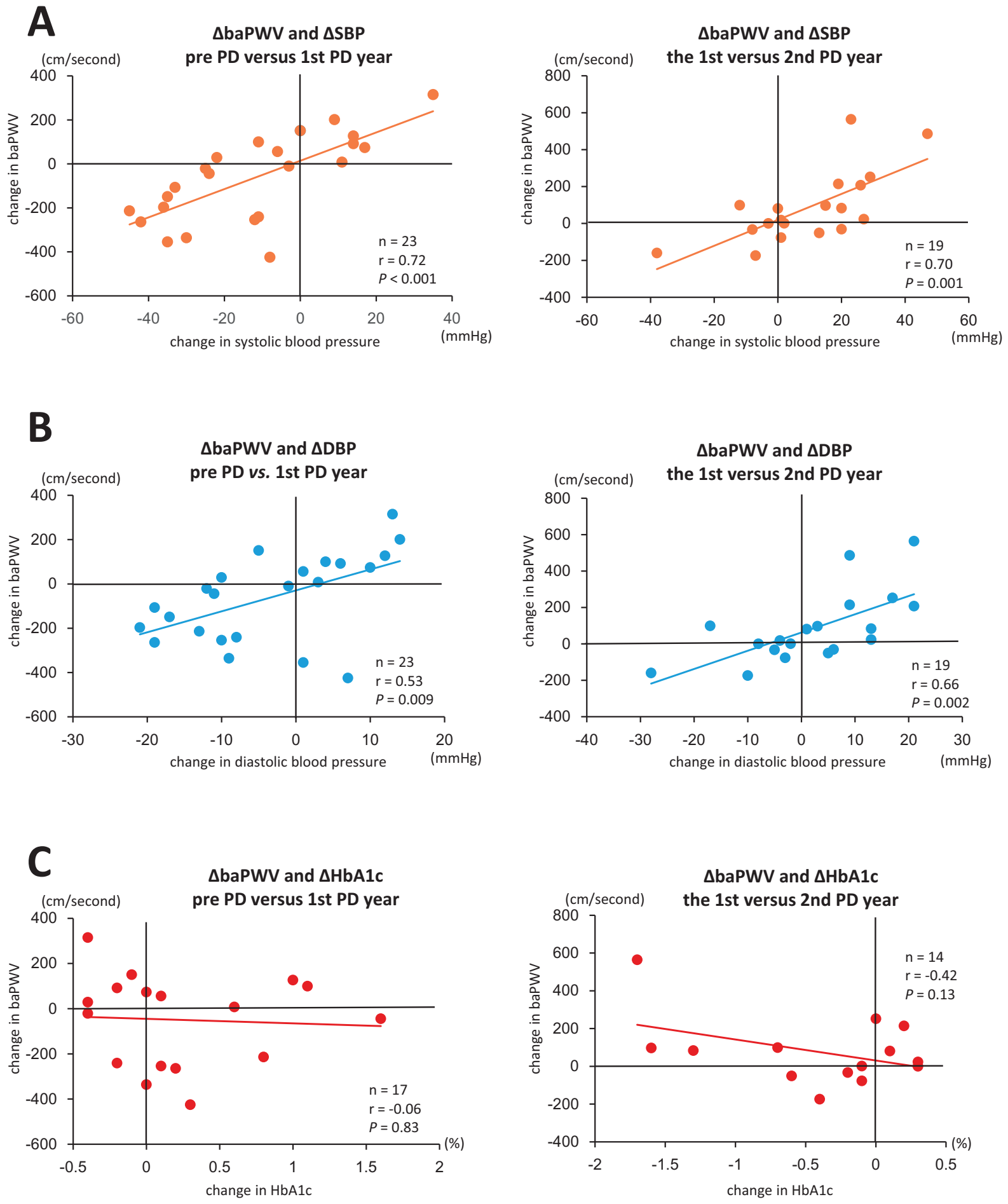

Figure 4. Univariate analysis of factors correlating with changes in baPWV. A: Relationship between changes in baPWV $(\Delta \mathrm{baPWV})$ and systolic BP ( $\Delta$ systolic BP). Positive associations were found during the first PD year (left) and the second PD year (right). B: Relationship between changes in baPWV $(\triangle \mathrm{baPWV})$ and diastolic BP $(\Delta$ diastolic BP). Positive correlations were observed during the first PD year (left) and continued to be the case during the second PD year (right). C: Relationship between changes in baPWV and HbAlc. There was no significant correlation during the first PD year (left) and the second PD year (right). Linear regression lines and Pearson correlation coefficients with their respective $P$ values are shown for each relationship. PD indicates peritoneal dialysis; BP, blood pressure; HbA1c, hemoglobin A1c; and baPWV, brachial-ankle pulse wave velocity. 
Table V. Hemodynamic Correlates of $\triangle \mathrm{baPWV}$

\begin{tabular}{lccc}
\hline \multicolumn{1}{c}{ Parameter } & $\beta$ coefficient & Standard error & $P$ \\
\hline Pre PD versus 1st PD year & & & \\
$\quad$ Change in systolic blood pressure & 0.72 & 1.37 & $<0.001$ \\
1st versus 2nd PD year & & & \\
$\quad$ Change in systolic blood pressure & 0.47 & 1.78 & 0.02 \\
$\quad$ Diastolic blood pressure at 2nd PD year visit & 0.45 & 2.77 & 0.02 \\
\hline
\end{tabular}

Multivariate model in pre PD versus 1st PD year was adjusted for change in diastolic blood pressure, systolic blood pressure (baseline prior to PD), and diastolic blood pressure (baseline), where multiple coefficient of determinant (adjusted $r^{2}$ ) was 0.49 . The relationships of variables were analyzed based on data from the first PD year $(n=23)$. Multivariate model in 1 st versus 2 nd year was adjusted for changes in diastolic BP, where multiple coefficient of determinant (adjusted $r^{2}$ ) was 0.59 . Standard regression coefficient $(\beta)$ is provided with its standard error and level of significance $(P)$. The relationships of variables were analyzed based on data from the second PD year $(n=19)$. PD indicates peritoneal dialysis; baPWV, brachial-ankle pulse wave velocity; and BP, blood pressure.
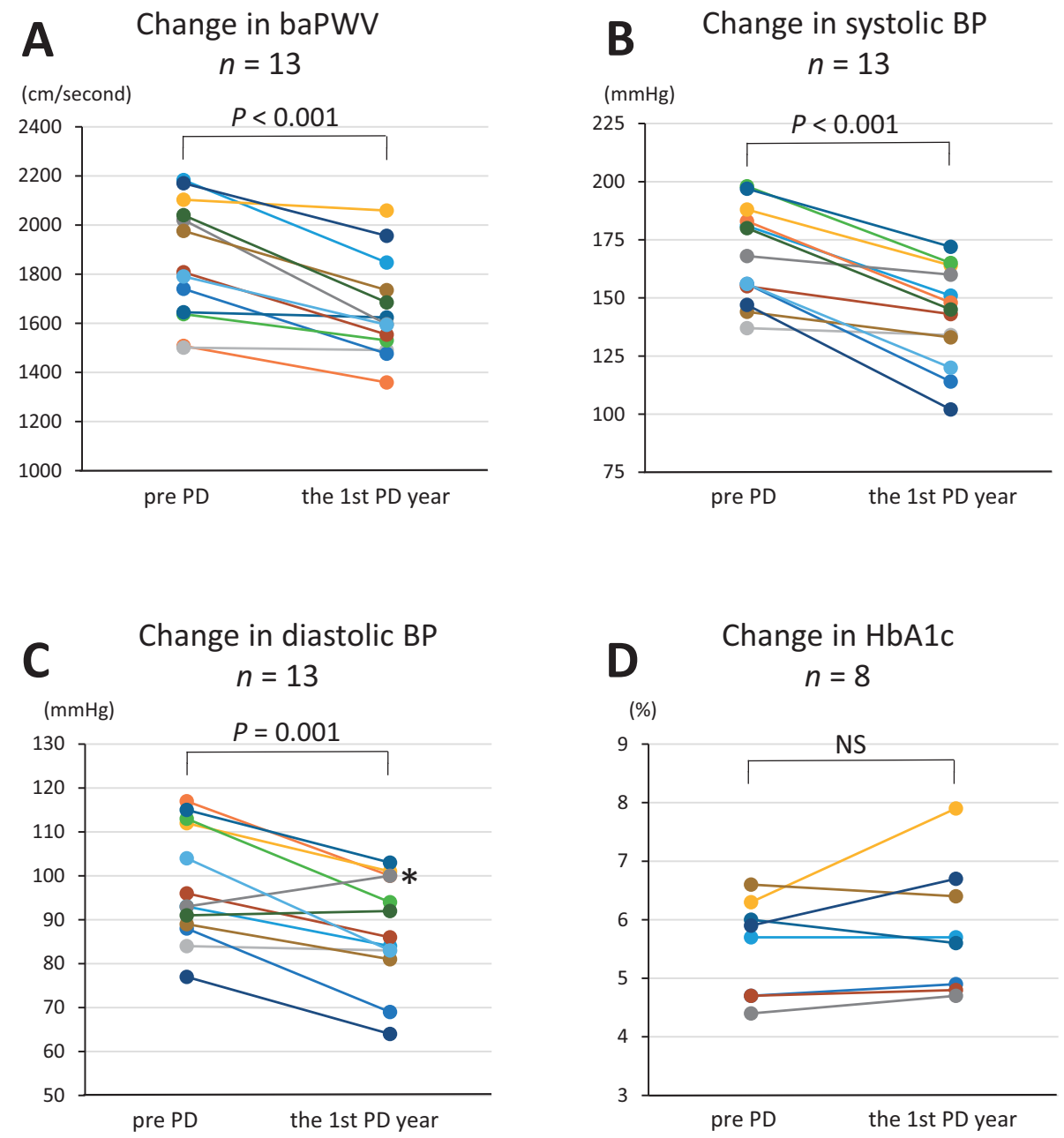

Figure 5. Longitudinal changes of baPWV and covariates during the first PD year. Thirteen out of 23 total PD patients who had a decreasing baPWV value over the first PD year $(n=13)$ were examined for their timecourse changes in systolic/diastolic BP and HbAlc profiles. The dots and lines are depicted by different colors to distinguish individual changes in the 13 patients. A: Changes in baPWV. baPWV values in the first year after starting PD (the first PD year) are relatively lower than those of base-line prior to PD initiation (pre-PD). B, C: Changes in hemodynamic parameters. All the patients who had a lowering baPWV value over the first PD year in A also displayed the concomitant decline in both systolic BP (B) and diastolic BP (C), with the exception of only one patient indicated by asterisk in C. D: Glycemic control levels. HbA1c did not change significantly over the first PD year $(n=8)$. PD indicates peritoneal dialysis; BP, blood pressure; HbA1c, hemoglobin A1c; baPWV, brachial-ankle pulse wave velocity; and NS, not significant. 
and hyperglycemia could accelerate the stiffness through mutually related, complementary mechanisms.

Our multivariate analyses demonstrated that increased cIMT of PD patients correlated with smoking and hypoalbuminemia. Cigarette smoking is widely accepted as a major risk factor for the development of CVD. It is thought that smoking induces an oxidative stress to activate the endothelial injury through accumulation of the macrophages and platelets. ${ }^{24)}$ This process is mediated by suppression of nitric oxide synthesis. Importantly, hypoalbuminemia was found to be as another independent correlate of cIMT. Relationship of low serum albumin and cIMT are in good agreement with previous observations that hypoalbuminemia was associated with inflammation and increased oxidative stress, the process which have been reported as major factors promoting arterial rigidity in dialysis patients. ${ }^{25}$ Since PD patients are prone to transperitoneal albumin loses and high-glucose dialysis exposure, there may be a complex association of endothelial dysfunction, inflammation, and hypoalbuminemia, each of which may mutually coactivate the atherogenic process. Further study is required to clarify to which extent these factors contribute to the CVD incidence on PD patients.

Several studies have assessed the impact of dialysis modality on the arterial stiffness. One previous study reported that, despite the similar stiffness levels (cfPWV) before dialysis between the two groups, PWVs for the HD patients are significantly higher than those for PD patients, when evaluated at one year after introduction of dialysis. ${ }^{26)}$ These results suggest less arterial damage in patients on PD than in patients on HD. Another study also confirmed that baPWV is higher in HD than in PD. ${ }^{27)}$ However, there are still some contradicting reports that failed to see any differences of PWV between PD and HD. ${ }^{28)}$ We consider that the conflicting results among these studies may reflect the differences in a) the way of PWV measurement (cfPWV vs baPWV), b) follow-up periods (short versus long term), and patient characteristics (age, duration dialysis, dialysis efficiency, control status of the background medical disorders, etc.). To further evaluate which dialysis modality is beneficial for the prevention of arterial stiffening, we will need to design the longitudinal study with larger number of the patients, which allows better comparison for the two subgroups (HD versus PD).

Because of the relatively small sample size in our study we may have missed some confounding variables that cause rigidification of arteries in PD patients. For instance, we did not see any association between stiffness and variables related to bone mineral metabolism. The role of calcification in the progression of arterial stiffness has been raised as a critical research question by KDIGO. ${ }^{29)}$ Arterial calcification usually develops in the intima or the media of muscular-type arteries, ${ }^{30,31)}$ and may take more time to manifest clinically. Diabetic microangiopathy also progresses at a much slower rate. PD modality may influence arterial rigidity and therefore it is important to compare the effects of volume, exchange methods (APD or CAPD), and contents of dialysates (glucose-based versus icodextrin).

In conclusion, our study demonstrated that baPWV is a useful clinical tool that could help earlier recognition and intervention of arterial stiffness in PD patients. BaPWV is associated with multiple but modifiable variables including BP, hyperglycemia, and proteinuria. It may therefore represent cumulative vascular risk derived from both hemodynamic and metabolic abnormalities. Optimization of BP as well as nutritional conditions (e.g., glucose and albumin levels) will be primary measure for ameliorating arterial stiffness during the initial stage of PD therapy. A large prospective study over a longer period will provide a broader scope of CVD risk in PD patients.

\section{Disclosures}

Conflicts of interest: The authors do not declare any conflict of interest.

\section{References}

1. Boutouyrie P, Fliser D, Goldsmith D, et al. Assessment of arterial stiffness for clinical and epidemiological studies: methodological considerations for validation and entry into the European Renal and Cardiovascular Medicine registry. Nephrol Dial Transplant 2014; 29: 232-9. (Review).

2. Hassan K, Hassan S, Anwar S, Zaher A, Edgem R, Hassan F. Predictors of left ventricular hypertrophy and their cutoffs in peritoneal dialysis patients. Int Heart J 2015; 56: 186-91.

3. Ter Avest E, Stalenhoef AF, de Graaf J. What is the role of noninvasive measurements of atherosclerosis in individual cardiovascular risk prediction? Clin Sci (Lond) 2007; 112: 507-16. (Review).

4. Mancia G, Fagard R, Narkiewicz K, et al. the Task Force for the Management of Arterial Hypertension of the European Society of Hypertension (ESH) and of the European Society of Cardiology (ESC). Eur Heart J 2013; 34: 2159-219.

5. Briet M, Pierre B, Laurent S, London GM. Arterial stiffness and pulse pressure in CKD and ESRD. Kidney Int 2012; 82: 388400. (Review).

6. Touboul PJ, Hennerici MG, Meairs S, et al. Advisory Board of the 3rd Watching the Risk Symposium 2004, 13th European Stroke Conference: Mannheim intima-media thickness consensus. Cerebrovasc Dis 2004; 18: 346-9. (Review).

7. Blacher J, Guerin AP, Pannier B, Marchais SJ, Safar ME, London GM. Impact of aortic stiffness on survival in end-stage renal disease. Circulation 2013; 99: 2434-9.

8. Kocyigit I, Sipahioglu MH, Orscelik O, et al. The association between arterial stiffness and fluid status in peritoneal dialysis patients. Perit Dial Int 2014; 34: 781-90.

9. Zhe XW, Tian XK, Chen W, et al. Association between arterial stiffness and peritoneal small solute transport rate. Artif Organs 2008; 32: 416-9.

10. Sipahioglu MH, Kucuk H, Unal A, et al. Impact of arterial stiffness on adverse cardiovascular outcomes and mortality in peritoneal dialysis patients. Perit Dial Int 2012; 32: 73-80.

11. Munakata M, Konno S, Miura Y, Yoshinaga K, J-TOPP Study Group. Prognostic significance of the brachial-ankle pulse wave velocity in patients with essential hypertension: final results of the J-TOPP study. Hypertens Res 2012; 35: 839-42.

12. Tikhonoff V, Casiglia E. Measuring regional arterial stiffness in patients with peripheral artery disease: innovative technology. Hypertens Res 2013; 36: 191-3.

13. Chou LP, Li CY, Hu SC. Work-related psychosocial hazards and arteriosclerosis. Int Heart J 2015; 56: 644-50.

14. Tomiyama H, Yamashina A, Arai T, et al. Influences of age and gender on results of noninvasive brachial-ankle pulse wave velocity measurement--a survey of 12517 subjects. Atherosclerosis 
2003; 166: 303-9.

15. Liu X, Sun N, Yu T, et al. The independent and joint association of blood pressure, serum total homocysteine, and fasting Serum glucose levels with brachial-ankle pulse wave velocity in Chinese hypertensive adults. Int Heart J 2016; 57: 627-33.

16. Yagi H, Sumino H, Yoshida K, et al. Biological antioxidant potential negatively correlates with carotid artery intima-media thickness. Int Heart J 2016; 57: 220-5.

17. Davenport A, Willicombe MK. Does diabetes mellitus predispose to increased fluid overload in peritoneal dialysis patients? Nephron Clin Pract 2010; 114: c60-6.

18. Jung JY, Hwang YH, Lee SW, et al. Factors associated with aortic stiffness and its change over time in peritoneal dialysis patients. Nephrol Dial Transplant 2010; 25: 4041-8.

19. Demirci MS, Gungor O, Kircelli F, et al. Impact of mean arterial pressure on progression of arterial stiffness in peritoneal dialysis patients under strict volume control strategy. Clin Nephrol 2012; 77: 105-13.

20. Tang M, Romann A, Chiarelli G, et al. Vascular stiffness in incident peritoneal dialysis patients over time. Clin Nephrol 2012; 78: 254-62.

21. Szeto CC, Kwan BC, Chow KM, Leung CB, Law MC, Li PK. Prognostic value of arterial pulse wave velocity in peritoneal dialysis patients. Am J Nephrol 2012; 35: 127-33.

22. Kuang DW, Li CL, Kuok UI, Cheung K, Lio WI, Xin J. Risk factors associated with brachial-ankle pulse wave velocity among peritoneal dialysis patients in Macao. BMC Nephrol 2012; 13: 143 .

23. Matsumae T, Abe Y, Murakami G, Ishihara M, Ueda K, Saito T. Determinants of arterial wall stiffness and peripheral artery occlusive disease in nondiabetic hemodialysis patients. Hypertens Res 2007; 30: 377-85.

24. Messner B, Bernhard D. Smoking and cardiovascular disease: mechanisms of endothelial dysfunction and early atherogenesis. Arterioscler Thromb Vasc Biol 2014; 34: 509-15. (Review).

25. Danielski M, Ikizler TA, McMonagle E, et al. Linkage of hypoalbuminemia, inflammation, and oxidative stress in patients receiving maintenance hemodialysis therapy. Am J Kidney Dis 2003; 42: 286-94.

26. Mimura T, Takenaka T, Kanno Y, Aoki H, Ohshima J, Suzuki H. Comparison of changes in pulse wave velocity in patients on continuous ambulatory peritoneal dialysis and hemodialysis one year after introduction of dialysis therapy. Adv Perit Dial 2005; 21: $139-45$.

27. Stróżecki P, Donderski R, Kardymowicz A, Manitius J. Comparison of arterial stiffness in end-stage renal disease patients treated with peritoneal dialysis or hemodialysis. Pol Arch Med Wewn 2012; 122: 33-9.

28. Chang JH, Yoon SJ, Han SH, et al. The impact of dialysis modality on arterial stiffness in patients with end-stage renal disease. Ren Fail 2010; 32: 947-53.

29. Moe S, Drüeke T, Cunningham J, et al. Kidney Disease: Improving Global Outcomes (KDIGO): Definition, evaluation, and classification of renal osteodystrophy: a position statement from Kidney Disease: Improving Global Outcomes (KDIGO). Kidney Int 2006; 69: 1945-53.

30. London GM, Guérin AP, Marchais SJ, Métivier F, Pannier B, Adda H. Arterial media calcification in end-stage renal disease: impact on all-cause and cardiovascular mortality. Nephrol Dial Transplant 2003; 18: 1731-40.

31. Koga S, Ikeda S, Nakata T, Kawano H, Abe K, Maemura K. Diverse findings in calcified thrombus between histopathology and in vivo imaging including intravascular ultrasound, optical coherence tomography, and angioscopy. Int Heart J 2015; 56: 6613. 